\title{
Causal Relationship between Obesity and Vitamin D Status: Bi-Directional Mendelian Randomization Analysis of Multiple Cohorts
}

\author{
Karani S. Vimaleswaran ${ }^{19}$, Diane J. Berry ${ }^{19}$, Chen Lu ${ }^{2}$, Emmi Tikkanen ${ }^{3,4}$, Stefan Pilz ${ }^{5,6}$, Linda T. Hiraki ${ }^{7}$, \\ Jason D. Cooper ${ }^{8}$, Zari Dastani ${ }^{9}$, Rui Li ${ }^{10}$, Denise K. Houston ${ }^{11}$, Andrew R. Wood ${ }^{12}$, Karl Michaëlsson ${ }^{13}$, \\ Liesbeth Vandenput ${ }^{14}$, Lina Zgaga ${ }^{15,16}$, Laura M. Yerges-Armstrong ${ }^{17}$, Mark I. McCarthy ${ }^{18,19,20}$, \\ Josée Dupuiss,21, Marika Kaakinen ${ }^{22}$, Marcus E. Kleber ${ }^{23}$, Karen Jameson ${ }^{24}$, Nigel Arden ${ }^{25,26,}$ \\ Olli Raitakari ${ }^{27}$, Jorma Viikari ${ }^{28}$, Kurt K. Lohman ${ }^{29}$, Luigi Ferrucci ${ }^{30}$, Håkan Melhus ${ }^{31}$, Erik Ingelsson ${ }^{32}$, \\ Liisa Byberg ${ }^{13}$, Lars Lind ${ }^{31}$, Mattias Lorentzon ${ }^{14}$, Veikko Salomaa ${ }^{4}$, Harry Campbell ${ }^{15}$, \\ Malcolm Dunlop ${ }^{33,34}$, Braxton D. Mitchell ${ }^{17}$, Karl-Heinz Herzig 22,35,36, Anneli Pouta ${ }^{37}$, Anna- \\ Liisa Hartikainen ${ }^{38}$, the Genetic Investigation of Anthropometric Traits (GIANT) consortium?, \\ Elizabeth A. Streeten ${ }^{17}$, Evropi Theodoratou ${ }^{15}$, Antti Jula ${ }^{4}$, Nicholas J. Wareham ${ }^{39}$, Claes Ohlsson ${ }^{14}$, \\ Timothy M. Frayling ${ }^{12}$, Stephen B. Kritchevsky ${ }^{11}$, Timothy D. Spector ${ }^{40}$, J. Brent Richards ${ }^{10,40}$, \\ Terho Lehtimäki ${ }^{41}$, Willem H. Ouwehand ${ }^{42,43,44}$, Peter Kraft' ${ }^{7}$ Cyrus Cooper ${ }^{24}$, Winfried März ${ }^{45,46}$, \\ Chris Power' ${ }^{1}$, Ruth J. F. Loos ${ }^{39}$, Thomas J. Wang ${ }^{47}$, Marjo-Riitta Järvelin ${ }^{22,48,49}$, John C. Whittaker ${ }^{50,51}$, \\ Aroon D. Hingorani ${ }^{52,53}$, Elina Hyppönen ${ }^{1 * 9}$
}

1 Centre for Paediatric Epidemiology and Biostatistics and MRC Centre of Epidemiology for Child Health, UCL Institute of Child Health, London, United Kingdom, 2 Department of Biostatistics, Boston University School of Public Health, Boston, Massachusetts, United States of America, 3 Institute for Molecular Medicine Finland FIMM, University of Helsinki, Helsinki, Finland, 4 National Institute for Health and Welfare, Helsinki, Finland, 5 Department of Internal Medicine, Division of Endocrinology and Metabolism, Medical University of Graz, Austria, $\mathbf{6}$ Department of Epidemiology and Biostatistics, EMGO Institute for Health and Care Research, VU University Medical Centre, Amsterdam, The Netherlands, 7 Program in Molecular and Genetic Epidemiology, Harvard School of Public Health, Boston, Massachusetts, United States of America, 8 Juvenile Diabetes Research Foundation/Wellcome Trust Diabetes and Inflammation Laboratory, Department of Medical Genetics, Cambridge Institute for Medical Research, University of Cambridge, Cambridge, United Kingdom, 9 Department of Epidemiology, Biostatistics and Occupational Health, Lady Davis Institute, Jewish General Hospital, McGill University, Montreal, Quebec, Canada, 10 Departments of Medicine, Human Genetics, Epidemiology and Biostatistics, Lady Davis Institute, Jewish General Hospital, McGill University, Montreal, Quebec, Canada, 11 Department of Internal Medicine, Section on Gerontology and Geriatric Medicine, Wake Forest School of Medicine, Winston Salem, North Carolina, United States of America, 12 Genetics of Complex Traits, Peninsula College of Medicine and Dentistry, University of Exeter, Exeter, United Kingdom, 13 Department of Surgical Sciences, Uppsala University, Uppsala, Sweden, 14 Center for Bone and Arthritis Research, Department of Internal Medicine, Institute of Medicine, University of Gothenburg, Gothenburg, Sweden, 15 Centre for Population Health Sciences, University of Edinburgh, Edinburgh, United Kingdom, 16 Andrija Stampar School of Public Health, Medical School University of Zagreb, Zagreb, Croatia, 17 University of Maryland School of Medicine, Division of Endocrinology, Baltimore, Maryland, United States of America, 18 Oxford Centre for Diabetes, Endocrinology and Metabolism, University of Oxford, Churchill Hospital, Headington, Oxford, United Kingdom, 19 Wellcome Trust Centre for Human Genetics, University of Oxford, Oxford, United Kingdom, 20 Oxford NIHR Biomedical Research Centre, Churchill Hospital, Headington, Oxford, United Kingdom, 21 National Heart, Lung, and Blood Institute's Framingham Heart Study, Framingham, Massachusetts, United States of America, 22 Institute of Health Sciences and Biocenter Oulu, University of Oulu, Oulu, Finland, 23 LURIC Study non-profit LLC, Freiburg, Germany and Mannheim Institute of Public Health, Social and Preventive Medicine, Mannheim Medical Faculty, University of Heidelberg, Mannheim, Germany, 24 MRC Lifecourse Epidemiology Unit, University of Southampton, Southampton, United Kingdom, 25 NIHR Musculoskeletal BRU, Botnar Research Centre, Oxford, United Kingdom, 26 MRC Lifecourse Epidemiology Unit, University of Southampton, Southampton, United Kingdom, 27 Research Centre of Applied and Preventive Cardiovascular Medicine, University of Turku and Department of Clinical Physiology and Nuclear Medicine, University of Turku and Turku University Hospital, Turku, Finland, 28 Department of Medicine, University of Turku and Turku University Hospital, Turku, Finland, 29 Department of Biostatistical Sciences, Division of Public Health Sciences, Wake Forest School of Medicine, Winston Salem, North Carolina, United States of America, 30 Clinical Research Branch, Harbor Hospital, Baltimore, Maryland, United States of America, 31 Department of Medical Sciences, Uppsala University, Uppsala, Sweden, 32 Department of Medical Epidemiology and Biostatistics, Karolinska Institutet, Stockholm, Sweden, 33 Colon Cancer Genetics Group and Academic Coloproctology, Institute of Genetics and Molecular Medicine, University of Edinburgh, United Kingdom, 34 MRC Human Genetics Unit Western General Hospital Edinburgh, United Kingdom, 35 Institute of Biomedicine, University of Oulu, Oulu, Finland, $\mathbf{3 6}$ Department of Psychiatry, Kuopio University Hospital, Kuopio, Finland, 37 Department of Public Health Science and General Practice, University of Oulu, Oulu, Finland, 38 Department of Obstetrics and Gynaecology and Public Health and General Practice, University of Oulu, Oulu, Finland, 39 MRC Epidemiology Unit, Institute of Metabolic Science, Addenbrooke's Hospital, Cambridge, United Kingdom, 40 Department of Twin Research and Genetic Epidemiology, King's College London, London, United Kingdom, 41 Department of Clinical Chemistry, Fimlab Laboratories, Tampere University Hospital and University of Tampere, Tampere, Finland, 42 Department of Haematology, University of Cambridge, United Kingdom, $\mathbf{4 3}$ Wellcome Trust Sanger Institute, Hinxton, Cambridge, United Kingdom, 44 NHS Blood and Transplant, Cambridge, United Kingdom, 45 Synlab Academy, Mannheim, Germany, $\mathbf{4 6}$ Mannheim Institute of Public Health, Social and Preventive Medicine, Mannheim Medical Faculty, University of Heidelberg, Mannheim, Germany, $\mathbf{4 7}$ Cardiology Division, Massachusetts General Hospital, Boston, Massachusetts, United States of America, $\mathbf{4 8}$ Department of Biostatistics and Epidemiology, School of Public Health, MRC-HPA Centre for Environment and Health, Imperial College, Faculty of Medicine, London, United Kingdom, 49 Department of Children, Young People and Families, National Institute for Health and Welfare, Oulu, Finland, 50 Department of Epidemiology and Population Health, London School of Hygiene and Tropical Medicine, London, United Kingdom, 51 Quantitative Sciences, GlaxoSmithKline, Stevenage, United Kingdom, 52 Genetic Epidemiology Group, Department of Epidemiology and Public Health, Division of Population Health, University College London, London, United Kingdom, $\mathbf{5 3}$ Division of Medicine, Centre for Clinical Pharmacology, University College London, London, United Kingdom 


\section{Abstract}

Background: Obesity is associated with vitamin D deficiency, and both are areas of active public health concern. We explored the causality and direction of the relationship between body mass index (BMI) and 25-hydroxyvitamin D [25(OH)D] using genetic markers as instrumental variables (IVs) in bi-directional Mendelian randomization (MR) analysis.

Methods and Findings: We used information from 21 adult cohorts (up to 42,024 participants) with 12 BMI-related SNPs (combined in an allelic score) to produce an instrument for BMI and four SNPs associated with 25(OH)D (combined in two allelic scores, separately for genes encoding its synthesis or metabolism) as an instrument for vitamin D. Regression estimates for the IVs (allele scores) were generated within-study and pooled by meta-analysis to generate summary effects. Associations between vitamin D scores and BMI were confirmed in the Genetic Investigation of Anthropometric Traits (GIANT) consortium $(n=123,864)$. Each $1 \mathrm{~kg} / \mathrm{m}^{2}$ higher BMI was associated with $1.15 \%$ lower $25(\mathrm{OH}) \mathrm{D}$ $\left(p=6.52 \times 10^{-27}\right)$. The BMI allele score was associated both with BMI $\left(p=6.30 \times 10^{-62}\right)$ and $25(\mathrm{OH}) \mathrm{D}(-0.06 \%[95 \% \mathrm{Cl}$ -0.10 to -0.02 ], $p=0.004)$ in the cohorts that underwent meta-analysis. The two vitamin $D$ allele scores were strongly associated with $25(\mathrm{OH}) \mathrm{D}\left(p \leq 8.07 \times 10^{-57}\right.$ for both scores) but not with BMI (synthesis score, $p=0.88$; metabolism score, $p=0.08$ ) in the meta-analysis. A $10 \%$ higher genetically instrumented BMl was associated with $4.2 \%$ lower $25(\mathrm{OH}) \mathrm{D}$ concentrations (IV ratio: -4.2 [95\% Cl -7.1 to -1.3$], p=0.005$ ). No association was seen for genetically instrumented $25(\mathrm{OH}) \mathrm{D}$ with $\mathrm{BMI}$, a finding that was confirmed using data from the GIANT consortium ( $p \geq 0.57$ for both vitamin $\mathrm{D}$ scores).

Conclusions: On the basis of a bi-directional genetic approach that limits confounding, our study suggests that a higher BMI leads to lower $25(\mathrm{OH}) \mathrm{D}$, while any effects of lower $25(\mathrm{OH}) \mathrm{D}$ increasing BMI are likely to be small. Population level interventions to reduce $\mathrm{BMI}$ are expected to decrease the prevalence of vitamin $\mathrm{D}$ deficiency.

Please see later in the article for the Editors' Summary.

Citation: Vimaleswaran KS, Berry DJ, Lu C, Tikkanen E, Pilz S, et al. (2013) Causal Relationship between Obesity and Vitamin D Status: Bi-Directional Mendelian Randomization Analysis of Multiple Cohorts. PLoS Med 10(2): e1001383. doi:10.1371/journal.pmed.1001383

Academic Editor: Cosetta Minelli, Centre for Biomedicine, EURAC, Italy

Received May 31, 2012; Accepted December 24, 2012; Published February 5, 2013

Copyright: (c) 2013 Vimaleswaran et al. This is an open-access article distributed under the terms of the Creative Commons Attribution License, which permits unrestricted use, distribution, and reproduction in any medium, provided the original author and source are credited.

Funding: The authors thank the British Heart Foundation (grant PG/09/023) and the UK Medical Research Council (MRC; grant G0601653) for funding this work. ADH is a British Heart Foundation Senior Research Fellow (Award FS05/125). EH is a Department of Health (UK) Public Health Career Scientist. This work was undertaken at the Centre for Paediatric Epidemiology and Biostatistics, which benefits from funding support from the MRC in its capacity as the MRC Centre of Epidemiology for Child Health. Research at the University College London Institute of Child Health and Great Ormond Street Hospital for Children NHS Trust benefits from R\&D funding received from the NHS Executive. No funding bodies had any role in study design, data collection and analysis, decision to publish, or preparation of the manuscript.

Competing Interests: LTH is currently supported by a Canada Institute of Research (CIHR) Fellowship award. CC has received honoraria and consulting fees from Amgen, Eli Lilly, Medtronic, Merck, Novartis, and Servier. WM is an employee of synlab laboratory services GmbH. Synlab offers vitamin D testing. TJW is on the scientific advisory board for Diasorin Inc and has received research support from them. JCW is $90 \%$ employed by GlaxoSmithKline (GSK) whilst maintaining a $10 \%$ appointment at London School of Hygiene \& Tropical Medicine (LSHTM), and holds GSK shares. All other authors declare that no competing interests exist.

Abbreviations: 25(OH)D, 25-hydroxyvitamin D; BMI, body mass index; GIANT, Genetic Investigation of Anthropometric Traits; IV, instrumental variable; MR, Mendelian randomization

*E-mail: e.hypponen@ucl.ac.uk

9 These authors contributed equally to this work.

- Membership of the Genetic Investigation of Anthropometric Traits (GIANT) consortium is provided in the Acknowledgments. 


\section{Introduction}

The prevalence of obesity has increased in the last two decades and it is presently the most common and costly nutritional problem [1-4]. In the United States, one-third of the population is affected by obesity, according to the National Health and Nutrition Examination Survey [5]. Despite a known genetic contribution, the increase in obesity prevalence has been largely attributed to lifestyle changes, which means that it is amenable to modification through public health and other interventions [6].

Vitamin D deficiency is another increasingly prevalent public health concern in developed countries [7-9], and there is evidence that vitamin D metabolism, storage, and action both influence and are influenced by adiposity. Observational studies have reported an increased risk of vitamin D deficiency in those who are obese; however, the underlying explanations and direction of causality are unclear [10]. Active vitamin D (1,25-dihydroxyvitamin D) may influence the mobilisation of free fatty acids from the adipose tissue [11]. In vitro experiments in rats have also shown that large doses of vitamin $\mathrm{D}_{2}$ lead to increases in energy expenditure due to uncoupling of oxidative phosphorylation in adipose tissues [12]. However, randomized controlled trials (RCTs) testing the effect of vitamin D supplementation on weight loss in obese or overweight individuals have provided inconsistent findings [13-15]. It has also been suggested that obesity could result from an excessive adaptive winter response, and that the decline in vitamin $\mathrm{D}$ skin synthesis due to reduced sunlight exposure contributes to the tendency to increase fat mass during the colder periods of the year $[16,17]$. However, vitamin D is stored in the adipose tissue and, hence, perhaps the most likely explanation for the association is that the larger storage capacity for vitamin $\mathrm{D}$ in obese individuals leads to lower circulating 25-hydroxyvitamin D [25(OH)D] concentrations, a marker for nutritional status [18].

In the Mendelian randomization (MR) approach, causality is inferred from associations between genetic variants that mimic the influence of a modifiable environmental exposure and the outcome of interest [19]. If lower vitamin D intake/status is causally related to obesity, a genetic variant associated with lower $25(\mathrm{OH}) \mathrm{D}$ concentrations should be associated with higher body mass index (BMI) (in proportion to the effect on $25(\mathrm{OH}) \mathrm{D})$. Conversely, if obesity leads to lower vitamin $\mathrm{D}$ status, then genetic variants associated with higher BMI should be related to lower $25(\mathrm{OH}) \mathrm{D}$ concentrations. The genetic associations, unlike the directly observed associations for vitamin D intake/status, should be less prone to confounding by lifestyle and socio-economic factors and be free from reverse causation as genotypes are invariant and assigned at random before conception [20]. The use of multiple SNPs to index the intermediate exposure of interest increases power and reduces the risk of alternative biological pathways (pleiotropy) affecting the observed associations between the genotype and the outcome [21,22].

In the present study, we investigated the relationship between BMI, a commonly used measure for monitoring the prevalence of obesity at the population level, and vitamin D status and we inferred causality by using genetic variants as instruments in bidirectional MR analyses. Meta-analysis included data from 21 studies comprising up to 42,024 individuals.

\section{Methods}

\section{Ethics Statement}

All participants provided written, informed consent, and ethical permission was granted by the local research ethics committees for all participating studies.

\section{Participants}

The collaboration investigating the association of vitamin D and the risk of cardiovascular disease and related traits (D-CarDia) consists of European ancestry cohorts from the United Kingdom (UK), United States (US), Canada, Finland, Germany, and Sweden. This study comprised a meta-analysis of directly genotyped and imputed SNPs from 21 cohorts totalling 42,024 individuals (Table 1). An expanded description of the participating studies is provided in the Text S2.

To replicate our findings on the association between the vitamin D-related SNPs and allele scores with BMI, we used the data from the genome-wide meta-analyses on BMI conducted as part of the Genetic Investigation of Anthropometric Traits (GIANT) consortium [23]. The GIANT meta-analyses consisted of 46 studies with up to 123,865 adults of European ancestry, including the 1958 British Birth Cohort, Framingham Heart study, Nurses' Health Study, Twins UK, UK Blood Services Common Control Collection, the Amish Family Osteoporosis Study, Health2000 GENMETS sub-sample, and Northern Finland Birth Cohort 1966, which were also part of the D-CarDia collaboration.

\section{Genotyping}

We selected 12 established BMI-related SNPs (fat mass and obesity-associated, [FTO]- rs9939609, melanocortin 4 receptor [MC4R]- rs17782313, transmembrane protein 18 [TMEM18]rs2867125, SH2B adaptor protein 1 [SH2B1]- rs7498665, brainderived neurotrophic factor $[B D \mathcal{N} F]-\mathrm{rs} 4074134$, potassium channel tetramerisation domain containing 15 [KCTD15]rs29941, ets variant 5 [ETV5]- rs7647305, SEC16 homolog B [SEC16B]- rs 10913469, Fas apoptotic inhibitory molecule 2 [FAIM2]- rs7138803, neuronal growth regulator 1 [NEGR1]rs3101336, mitochondrial carrier 2 [MTCH2]- rs10838738, and glucosamine-6-phosphate deaminase 2 [GNPDA2]- rs10938397) for our analysis based on the study by Li et al. [24] and previously published genome-wide association studies for obesity-related traits $[23,25,26]$. The four vitamin D-related SNPs (DHCR7rs12785878, CYP2R1-rs10741657, GC-rs2282679, and CYP24A1rs6013897) were chosen on the basis of the recent genome-wide association study on $25(\mathrm{OH}) \mathrm{D}$ [27]. The studies that did not have genotyped data analysed imputed or proxy $\operatorname{SNPs}\left(\mathrm{r}^{2}=1\right)$ as available (with a call threshold of 0.9 for the SNPs imputed with Impute; for those imputed with MACH, a call threshold of 0.8 was used) [28]. The genetic data for most studies were obtained from genome-wide association platforms, but for some studies, variants were genotyped de novo (MRG Ely, the Canadian Multicentre Osteoporosis Study, the Hertfordshire cohort study) or obtained through metabochip custom array (MRC Ely). Five studies did not have all the BMI-related SNPs (Framingham Heart Study [one missing SNP], Hertfordshire cohort study [three missing SNPs], InCHIANTI [two missing SNPs], PIVUS [two missing SNPs], and ULSAM [three missing SNPs]) and were still included in the BMI allele score analysis. Table $\mathrm{S} 1$ shows the minor allele frequencies for the BMI and vitamin D SNPs that were included in the analysis. A detailed description of the genotyping methods is provided in Text S2.

\section{Statistical Analysis}

Analyses in each study were performed according to a standardized analysis plan. When used as outcome variables, $25(\mathrm{OH}) \mathrm{D}$ and $\mathrm{BMI}$ were natural $\log$ transformed to be more closely approximated by normal distributions. If multiplied by 100 , coefficients from linear regression models with $\ln$ transformed outcomes can be interpreted as the percentage difference in the outcome [29]. Models with BMI as an outcome were adjusted for 


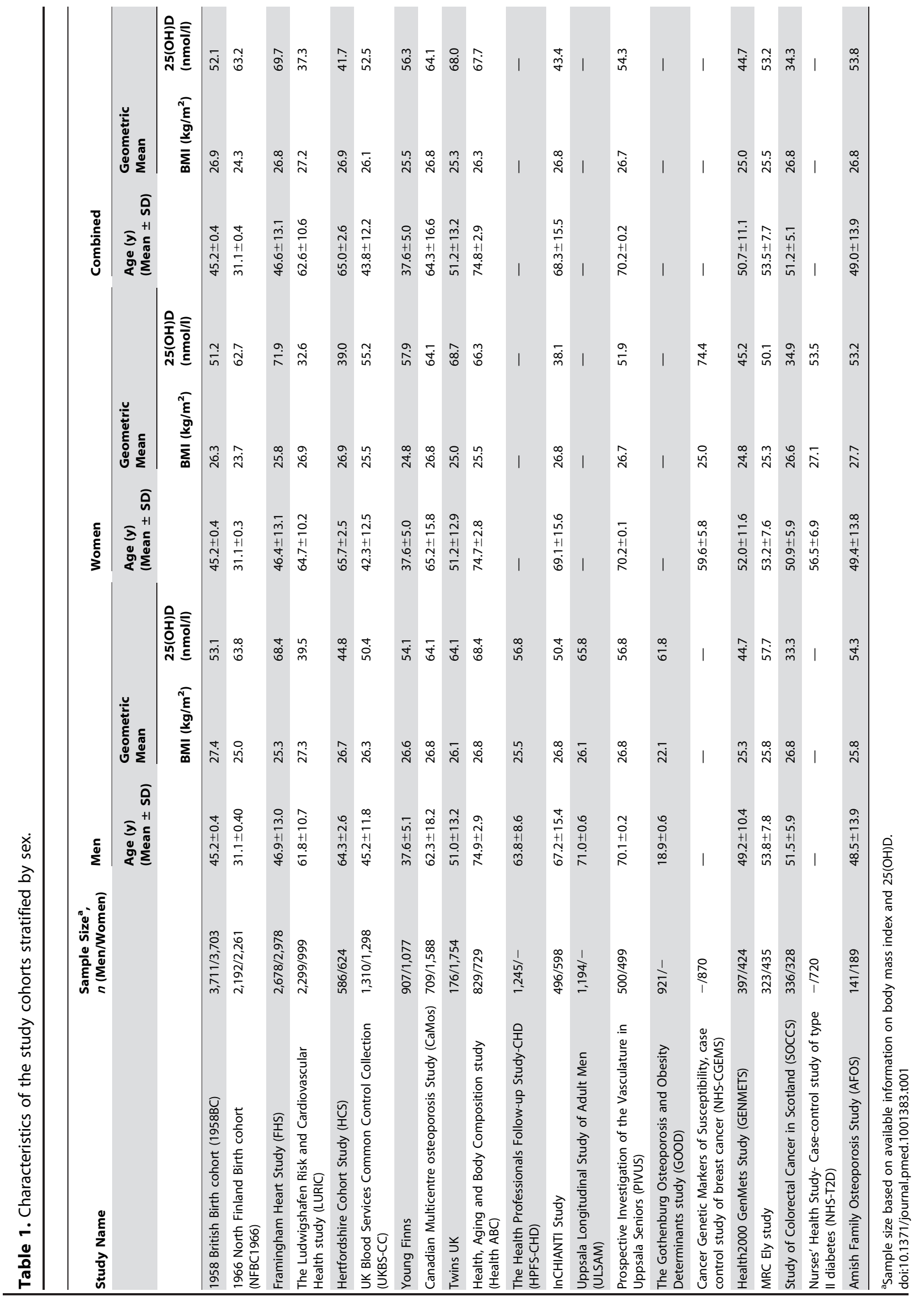


age, sex, geographical site, and/or principal components from population stratification analysis (depending on data available); models with $25(\mathrm{OH}) \mathrm{D}$ as the outcome were additionally adjusted for month of blood sample collection (as a categorical variable) to account for seasonal variation and laboratory batch, where relevant. To assess the BMI relationship with $25(\mathrm{OH}) \mathrm{D}$ and vice versa, each study ran linear regression models adjusting for the covariates listed for each outcome, and the models were repeated stratifying by sex.

For the BMI SNPs, the effect allele was the BMI raising allele as established by Speliotes et al. [23]. We created a weighted score in each study [30], by multiplying each SNP (coded as $0-2$ ) by a weight based on its effect size with BMI in the meta-analysis by Speliotes et al. [23]. The weighted BMI allele score was rescaled over the sum of weights for the available SNPs in each study to facilitate interpretation [30]. For the vitamin D SNPs, the effect allele was the $25(\mathrm{OH}) \mathrm{D}$ lowering allele as established by the SUNLIGHT Consortium [27]. As external weights were not available and the use of internal weights could bias the instrumental variable (IV) results [31], we performed an unweighted allele score analysis for the vitamin D SNPs. Vitamin D SNPs were used to form two separate allele scores [32]: a "synthesis" allele score, created by summing the risk alleles in DHCR7 and CYP2R1, and a "metabolism" allele score, created by summing the risk alleles in $G C$ and CYP24A1 (Figure S1). Synthesis allele score was not created for the LURIC study (one missing $\mathrm{SNP}$ ) and both synthesis and metabolism allele scores were not created for the MRG Ely study (two missing SNPs). The synthesis allele score included the SNPs that contribute directly to the production of $25(\mathrm{OH}) \mathrm{D}$, and hence, for which the association with the outcome can be readily estimated based on the magnitude of the association between the score and 25(OH)D [32]. All analyses were done separately for the "metabolism" SNPs that are involved in the clearance or transport of $25(\mathrm{OH}) \mathrm{D}$ (with possible influences on bioavailability [33]) as the quantification of the association with the outcome based on the observed SNP-25(OH)D association is more difficult [32]. We also evaluated the joint contribution of synthesis and metabolism scores on BMI by including both vitamin $\mathrm{D}$ scores as separate variables in a multiple regression model. To examine the strength of the allele scores as instruments, the $\mathrm{F}$-statistic was approximated from the proportion of variation in the respective phenotype $\left(\mathrm{R}^{2}\right)$ explained by the allele score, $[\mathrm{F}$ stat $\left.=\left(\mathrm{R}^{2} \times(n-2)\right) /\left(1-\mathrm{R}^{2}\right)\right][34]$.

To confirm our findings on the association between the vitamin D-related SNPs and allele scores with BMI in a larger sample, we used the summary statistics for the four vitamin D-related SNPs from the GIANT consortium. These SNPs were combined into synthesis and metabolism allele scores using an approximation method as previously described [35]. The individual SNP association with $\mathrm{BMI}$ is then weighted according to its predefined effect size and meta-analysed using the inverse-variance method with the other SNPs in the score [35]. The formal MR analyses to estimate the possible causal effect of BMI on 25(OH)D (and vice versa) were done using the IV ratio method $[20,36]$. To estimate the IV ratio for the BMI effect on $25(\mathrm{OH}) \mathrm{D}$, the meta-analysed association of the BMI allele score with $25(\mathrm{OH}) \mathrm{D}$ was divided by the association of BMI allele score with BMI. The variance for the IV ratio was estimated using a Taylor expansion [36]. The corresponding calculation was done to establish the $25(\mathrm{OH}) \mathrm{D}$ effect on BMI, with the IV ratio method applied separately for the two vitamin $\mathrm{D}$ allele scores. The joint contribution of the two vitamin $\mathrm{D}$ scores on $\mathrm{BMI}$ was assessed by multivariate metaanalysis [37], which incorporated the covariance matrix as estimated by study specific analyses.
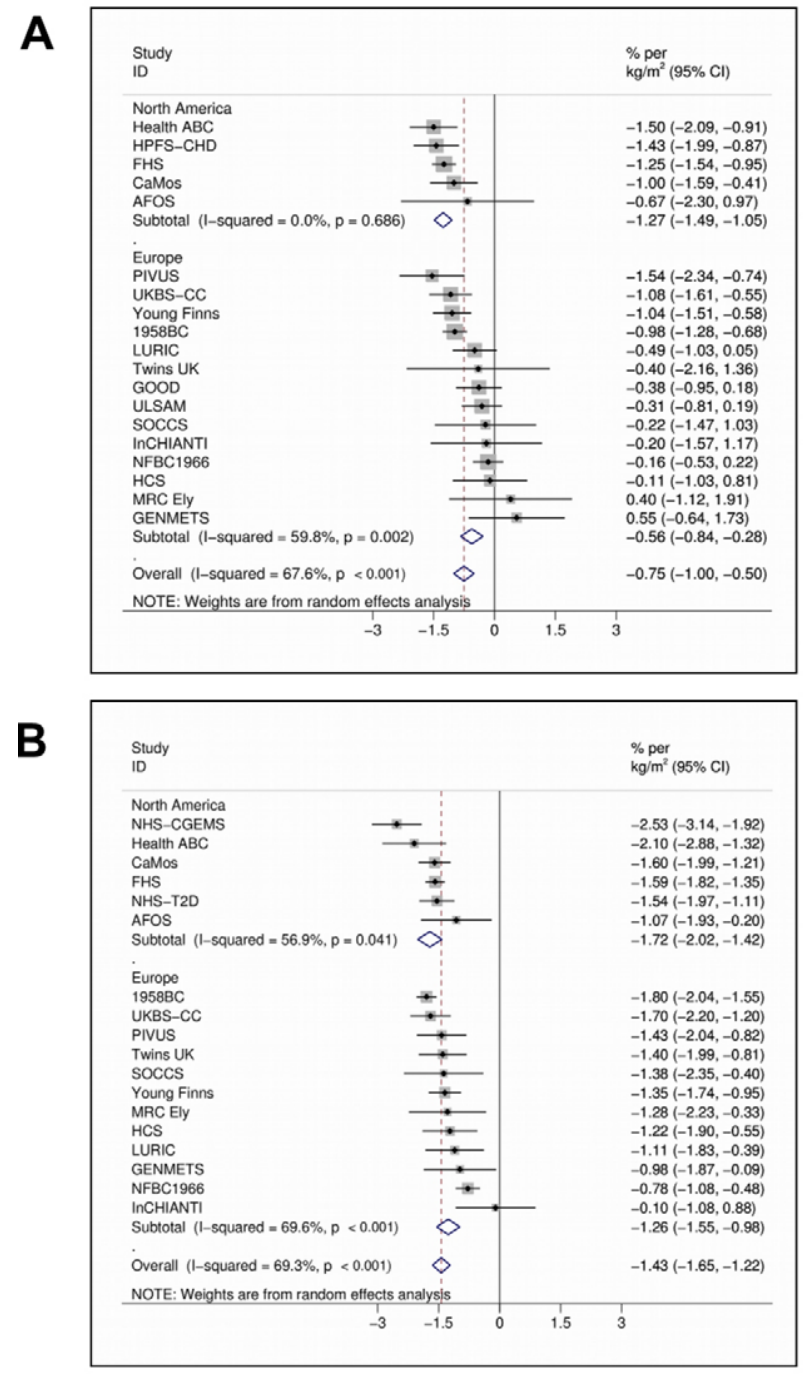

Figure 1. Random effects meta-analysis of the BMI association with 25(OH)D in men (A) $(n=20,950)$ and women (B) ( $n=\mathbf{2 1}, \mathbf{0 7 4 )}$. 95\% confidence intervals given by error bars. doi:10.1371/journal.pmed.1001383.g001

In the presence of heterogeneity of association between the studies, random effects meta-analyses [38] were run, otherwise fixed effects models were used. Univariate meta-regression models were run to assess differences in the observed associations by study level factors of sex, average BMI $\left(\mathrm{BMI} \leq 25 \mathrm{~kg} / \mathrm{m}^{2}\right.$ versus $>25 \mathrm{~kg}$ / $\left.\mathrm{m}^{2}\right)$, the average age of participants $(\leq 40,41-60$, and $\geq 61$ y old), continent (North America versus Europe), and vitamin D assay (radio-immunoassay, enzyme-linked radio-immunoassay, and mass spectrometry). Power calculations for IV regression were performed by simulation [32] on the basis of associations observed between the phenotypes and their genetic proxies. For comparability across instruments/outcomes, power was determined for $0.02 \log$ unit increase/decrease by decile, approximately corresponding to the association observed between BMI and 25(OH)D. To evaluate the ability to detect weaker effects on BMI using the synthesis and metabolism scores, power was also calculated for a $50 \%$ weaker effect (0.01 log unit increase/decrease). All metaanalyses and power calculations were performed at the Institute of Child Health (University College London, London) using STATA version 12 [39]. 


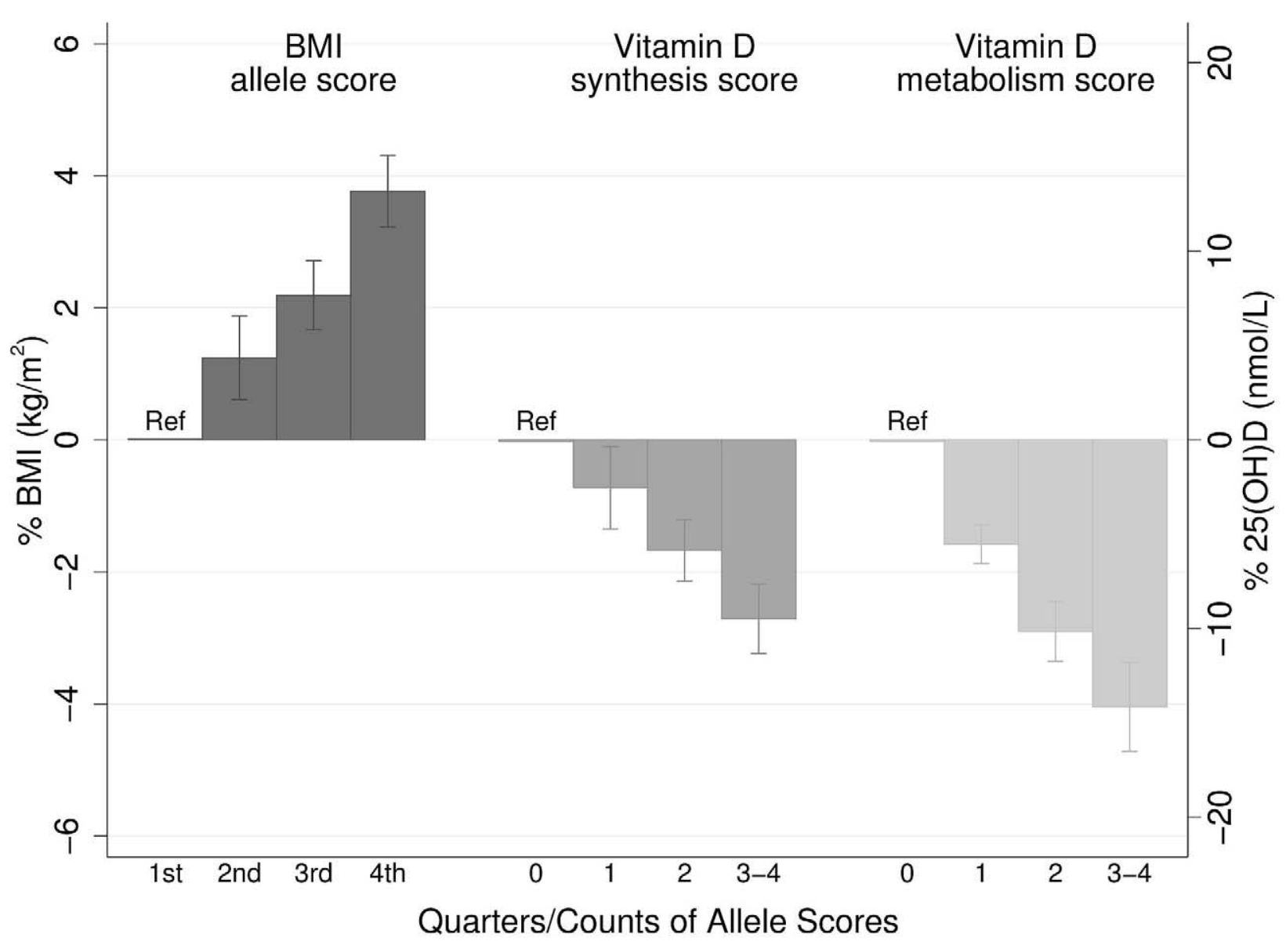

Figure 2. Meta-analysis of the BMI allele score association with BMI $(n=32,391)$, and the vitamin $\mathrm{D}$ synthesis $(n=35,873)$ and metabolism $(n=38,191)$ allele score association with $\mathbf{2 5}(\mathrm{OH}) \mathrm{D} .95 \%$ confidence intervals given by error bars. doi:10.1371/journal.pmed.1001383.g002

\section{Results}

\section{Phenotypic Association between BMI and 25(OH)D Concentrations}

In the meta-analyses of 21 studies, each unit $\left(\mathrm{kg} / \mathrm{m}^{2}\right)$ increase in BMI was associated with $1.15 \% \quad(95 \%$ CI $0.94 \%-1.36 \%$, $\left.p=6.52 \times 10^{-27}\right)$ lower concentrations of $25(\mathrm{OH}) \mathrm{D}$ after adjusting for age, sex, laboratory batch, month of measurement, and principal components. The inverse association between BMI and $25(\mathrm{OH}) \mathrm{D}$ was stronger among the studies from North America than those from Europe $(-1.58 \%[-1.81 \%$ to $-1.36 \%]$, $p=1.01 \times 10^{-43}$ versus $-0.91 \% \quad[-1.18 \%$ to $-0.64 \%]$, $\left.p=4.55 \times 10^{-11} ; p_{\text {meta-regression }}=0.004\right)$ and for women than men $\left(-1.43 \%[-1.65 \%\right.$ to $-1.22 \%], p=1.13 \times 10^{-38}$ versus $-0.75 \%$ $[-1.00 \%$ to $-0.50 \%], p=3.89 \times 10^{-9} ; p_{\text {meta-regression }}=4.10 \times 10^{-4}$ ) while no variation was seen by average age $\left(p_{\text {meta-regression }}=0.78\right)$ or $\mathrm{BMI}\left(p_{\text {meta-regression }}=0.48\right)($ Figure $1 \mathrm{~A}$ and $1 \mathrm{~B})$.

\section{Evaluation of Causal Association Using MR Approach}

The BMI allele score created from the 12 BMI-related SNPs showed a positive dose-response association with BMI (per unit increase $\left.0.14 \% \quad[0.12 \%-0.16 \%], p=6.30 \times 10^{-62}\right)$, and both vitamin $\mathrm{D}$ allele scores showed the expected strong associations with $25(\mathrm{OH}) \mathrm{D}$ (per allele in synthesis score: $-3.47 \%[-3.90 \%$ to $-3.05 \%], \quad p=8.07 \times 10^{-57} ;$ metabolism allele score: $-5.38 \%$
$[-5.84 \%$ to $-4.93 \%], p=1.07 \times 10^{-118}$ ) (Figures 2, S2, and S3). The BMI allele score was also associated with $25(\mathrm{OH}) \mathrm{D}$ concentrations (per unit increase $-0.06 \%, \quad[-0.10 \%$ to $-0.02 \%$ ], $p=0.004$ ) (Figure 3), while no association with BMI was seen for either the vitamin D synthesis or metabolism allele scores (per allele in synthesis score: $0.01 \%$ [ $-0.17 \%$ to $0.20 \%]$, $p=0.88$, metabolism allele score: $0.17 \%[-0.02 \%$ to $0.35 \%]$, $p=0.08]$ ) (Figure 4A and 4B). Analyses of joint effects by synthesis and metabolism scores provided no evidence for an association between 25(OH)D and BMI (per allele in synthesis score $-0.03 \%$ $[-0.23 \%$ to $0.16 \%]$ and metabolism score $0.17 \%[-0.04 \%$ to $0.37 \%]$, joint contribution $p=0.26$ ).

In the analyses to establish the direction and causality of BMI$25(\mathrm{OH}) \mathrm{D}$ association by the use of the IV ratio, BMI was associated with $25(\mathrm{OH}) \mathrm{D}$ : each $10 \%$ increase in $\mathrm{BMI}$ lead to a $4.2 \%$ decrease in $25(\mathrm{OH}) \mathrm{D}$ concentrations $(-7.1 \%$ to $-1.3 \%$; $p=0.005)$. However, the IV ratio analyses provided little evidence for a causal effect of $25(\mathrm{OH}) \mathrm{D}$ on BMI $(p \geq 0.08$ for both). We have summarised the coefficients for the MR analyses in Table 2.

The lack of association of the vitamin D allele scores with BMI was further confirmed using the GIANT consortium including 123,864 individuals in 46 studies [23]: neither the synthesis nor the metabolism allele score showed any evidence for an association with BMI ( $p \geq 0.57$ for both) (Table 3). 


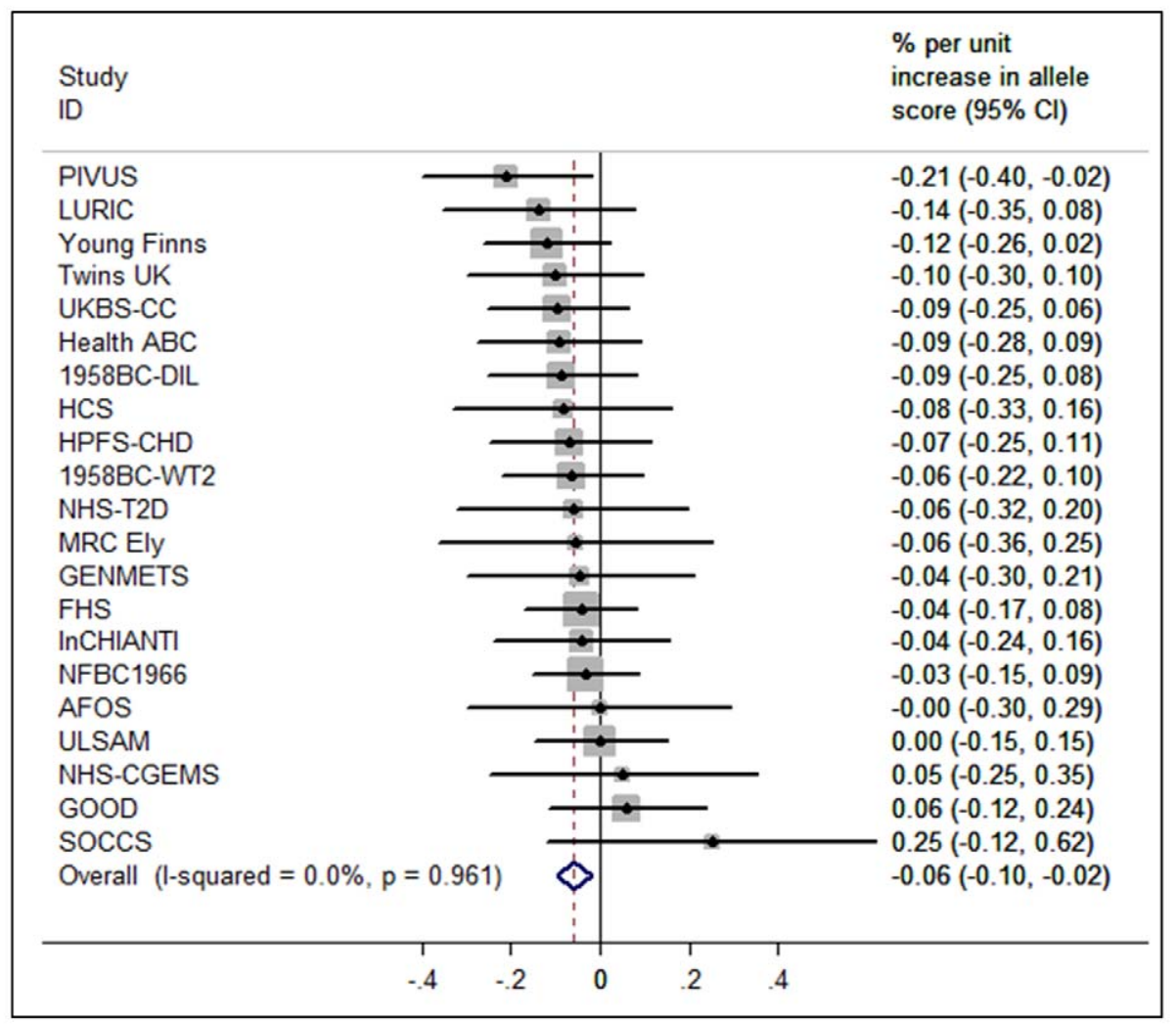

Figure 3. Meta-analysis of the BMI allele score association with $\mathbf{2 5}(\mathbf{O H}) \mathrm{D}(\boldsymbol{n}=\mathbf{3 1 , 1 2 0})$. 95\% confidence intervals given by error bars. doi:10.1371/journal.pmed.1001383.g003

\section{Additional Analyses}

Validation of the genetic instruments. The BMI SNPs and the vitamin D SNPs were all individually associated with BMI and $25(\mathrm{OH}) \mathrm{D}$, respectively (Figures $\mathrm{S} 4$ and S5). The exception was KCTD15 SNP, which despite previous evidence for an association [25], was not associated with BMI in our metaanalyses. Across the studies, the 12 BMI SNPs combined as the BMI allele score explained $0.97 \%$ of the variation in BMI (Fstatistic $=316 ; n=32,391)$. The synthesis allele score explained $0.64 \%($ F-statistic $=230 ; n=35,873)$ and the metabolism allele score $1.26 \%$ ( $\mathrm{F}$ statistic $=489 ; n=38,191)$ of the variation in $25(\mathrm{OH}) \mathrm{D}$. There was no evidence for variation in the BMI allele score-BMI association by continent $\left(p_{\text {meta-regression }}=0.15\right)$ or BMI $\left(p_{\text {meta-regression }}=0.83\right)$. However, the BMI allele score-BMI association was slightly weaker in studies with older compared to younger participants $(-0.03 \% \quad[-0.05 \%$ to $-0.002 \%]$, $\left.p_{\text {meta-regression }}=0.03\right)$. The vitamin $\mathrm{D}$ allele score $-25(\mathrm{OH}) \mathrm{D}$ association did not vary by age, BMI, continent, or assay ( $p_{\text {meta-regression }} \geq 0.09$ for all comparisons).

Evaluation of the genetic outcome associations. Of the 12 individual BMI SNPs, the SNP for FTO was the only one that showed evidence of a univariate association with $25(\mathrm{OH}) \mathrm{D}$ $(p=0.050)$ (Figure S6). None of the four 25(OH)D SNPs were individually associated with BMI $(p \geq 0.10)$ (Figure S7). The lack of association of the four vitamin D SNPs with BMI was further confirmed using the summary data from the GIANT consortium $(p>0.30$ for all the SNPs) (Table 3).

The association between BMI allele score and 25(OH)D did not vary by study level factors, including age $\left(p_{\text {meta-regression }}=0.40\right), \mathrm{BMI}$ $\left(p_{\text {meta-regression }}=0.18\right)$, continent of study $\left(p_{\text {meta-regression }}=0.78\right)$, or vitamin D assay $\left(p_{\text {meta-regression }}=0.23\right)$. Similarly, there was no evidence for variation in the vitamin $\mathrm{D}$ allele score-BMI association by age ( $p_{\text {meta-regression }} \geq 0.25$ for both scores), or continent ( $p_{\text {meta-regression }} \geq 0.50$ for both scores). There was also no strong evidence for variation in the vitamin $\mathrm{D}$ allele score-BMI association by average BMI of the study $\left(\leq 25 \mathrm{~kg} / \mathrm{m}^{2}\right.$ versus $\geq 25 \mathrm{~kg} / \mathrm{m}^{2}$ ), although for the synthesis score the meta-regression coefficient was of borderline significance $\left(p_{\text {meta-regression }}=0.053\right.$, Figure S8; $p_{\text {meta-regression }}=0.78$ for metabolism score).

Power comparison. Illustrative power calculations are presented in Figure S9. In theory, we had greater power to detect an association between 25(OH)D and BMI using the metabolism score as an instrument, compared with an equal sized association between $\mathrm{BMI}$ and 25(OH)D using the BMI risk score. However, if the size of the association between $25(\mathrm{OH}) \mathrm{D}$ and $\mathrm{BMI}$ was only half that seen between BMI and $25(\mathrm{OH}) \mathrm{D}$, our study would not have been adequately powered even with the inclusion of the GIANT results.

\section{Discussion}

Obesity, and perhaps vitamin D deficiency, are among the most important modifiable risk factors for a number of chronic diseases. Obesity and vitamin D status are known to be associated but the direction of the association and whether it is causal has been uncertain. We have presented genetic evidence that higher BMI leads to lower vitamin D status. Conversely, our analyses provided no evidence for a causal role of vitamin $\mathrm{D}$ in the development of obesity, although our study was not powered to detect very small effects. These results suggest that although increases in vitamin D status are not likely to help with weight regulation, increased risk 
A

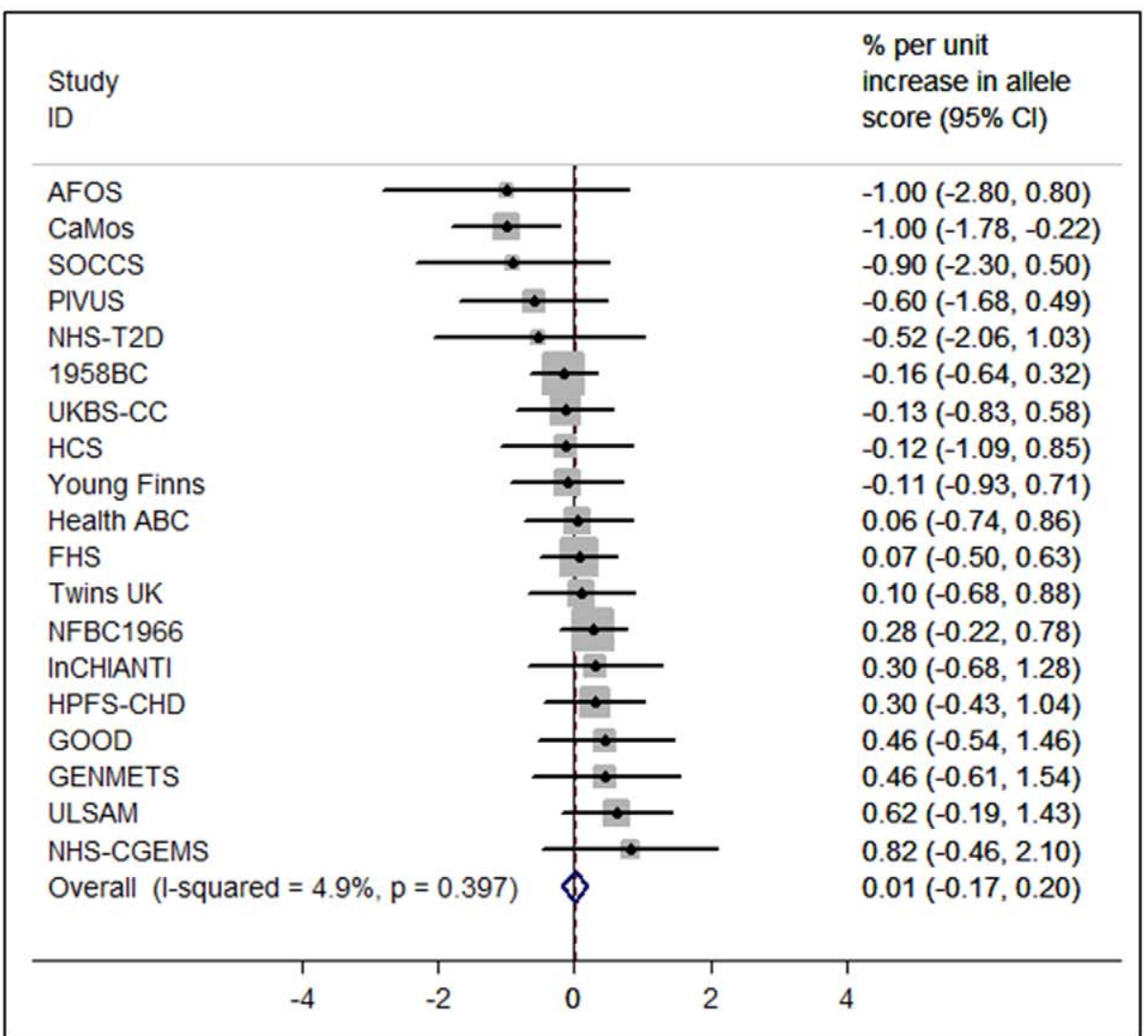

B

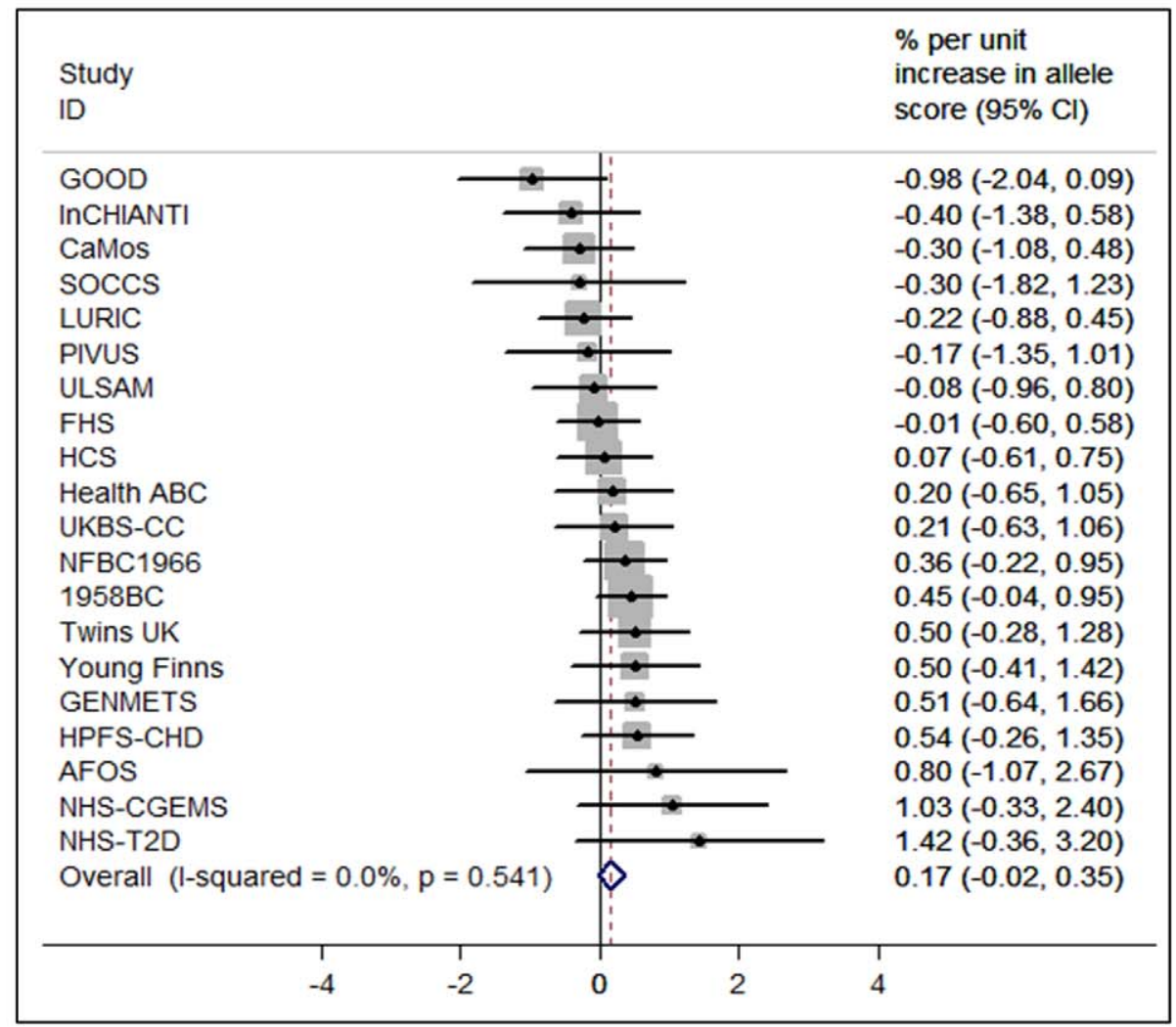


Figure 4. Meta-analysis of the synthesis allele score association with BMI $(A)(n=36,553)$ and the metabolism allele score association with BMI (B) $(n=40,367)$. 95\% confidence intervals given by error bars.

doi:10.1371/journal.pmed.1001383.g004

of vitamin $\mathrm{D}$ deficiency could contribute to the adverse health effects associated with obesity.

The association between obesity and vitamin D status was remarkably consistent across the different populations included in our meta-analyses, being apparent both in men and in women, and in the young and older cohorts alike. Interestingly, the association between obesity and $25(\mathrm{OH}) \mathrm{D}$ concentrations appeared stronger for populations in North America compared to Europe, possibly reflecting differences in the distribution of BMI across the continents. Recent intervention studies have shown that obese individuals need higher vitamin $\mathrm{D}$ dosages than lean individuals to achieve the same $25(\mathrm{OH}) \mathrm{D}$ concentrations $[40,41]$. Given that North America has one of the highest rates of obesity in the world [42], our study highlights the importance of considering obesity as a risk factor for vitamin D deficiency with implications on the dosage requirements and possible targeting of relevant health promotion strategies.

The lack of any suggestion for an association between the vitamin D SNPs and BMI in the GIANT consortium $(n=123,864)$ alongside our own large meta-analyses provides a strong case against linear increases in $25(\mathrm{OH}) \mathrm{D}$ having a substantive influence on BMI. This conclusion is in accordance with a recent study on Chinese women $(n=7,000)$, which also failed to observe evidence for an association with BMI for genetic variants in the vitamin D pathway [43]. Although a recent RCT $(n=77)$ suggested greater loss in fat mass for women receiving vitamin D [15], previous trials have failed to show any evidence for an effect despite larger treatment groups $(n=200-445)$, use of higher vitamin D dosages, and equal duration of treatment (12 mo) [13,14]. Dilution related to the greater volume of distribution has been recently proposed as the most likely explanation for the lower $25(\mathrm{OH}) \mathrm{D}$ concentrations in obese individuals [44]. In that study, no evidence was found for reduced bioavailability through increased sequestration of vitamin $\mathrm{D}$ in the adipose tissue, which had previously been suggested to contribute to the low $25(\mathrm{OH}) \mathrm{D}$ concentrations in obesity [18]. In contrast, intact parathyroid hormone (iPTH) levels [45], which stimulate the 1- $\alpha$-hydroxylase (CYP27B1) enzyme that converts $25(\mathrm{OH}) \mathrm{D}$ to 1,25 -dihydroxyvitamin $\mathrm{D}$ (the active hormonal form), have been found to be elevated in obesity [46], which could to some extent also contribute to the lower $25(\mathrm{OH}) \mathrm{D}$ concentrations in obese individuals. It is also possible that differences in lifestyle could contribute to lower $25(\mathrm{OH}) \mathrm{D}$ concentrations in obese compared to normal weight individuals, although the association between obesity and low $25(\mathrm{OH}) \mathrm{D}$ concentrations has been found to only modestly attenuate after adjustment for vitamin D-related lifestyle and dietary factors [9].
The main strengths of this study are the large sample size and the individual level population-based data from North America and Europe. We used a bi-directional MR approach to investigate the causal directions between obesity and vitamin D deficiency, observing evidence for reductions in $25(\mathrm{OH}) \mathrm{D}$ by BMI but not vice versa. However, based on the biological pathways proposed, a possible effect of $25(\mathrm{OH}) \mathrm{D}$ on BMI could be expected to be weaker than the effect of BMI on 25(OH)D. Despite including data from the large GIANT consortium to narrow the range of effects compatible with the data, we are unable to exclude very small effects. Furthermore, while the MR approach enables the approximation of life-long differences in average concentrations, with genetic markers it is not possible to examine the influences arising from the extremes of non-linear distributions [20]. Consequently, we cannot discount a possible effect of severe vitamin D deficiency on BMI due to evidence of non-linearity seen in some studies [47]. In contrast, associations between BMI and $25(\mathrm{OH}) \mathrm{D}$ within levels in the obesity range were consistently linear in studies included in our analyses (unpublished data), hence the observed association between higher BMI and lower $25(\mathrm{OH}) \mathrm{D}$ is likely to be informative in the context of obesity.

One of the methodological challenges of the MR approach relates to the large sample size requirement, arising from the availability of relatively weak instruments for most exposures [22,31]. This aspect of the MR approach is also reflected in our study, notably in the relatively small amount of variation explained by all the instruments used. We used the IV ratio method on metaanalyzed coefficients since all studies were not able to share individual level participant data. This method assumes linear relationships and may have less power to detect an effect than other IV methods [48]. However, as shown by the clear outcome of these analyses, we were able to overcome these issues by combining several cohorts with comparable information, allowing us to achieve the large numbers required (maximum $n=42,024$ ) [31]. To confirm the lack of association between vitamin D-related genetic variations and BMI, we were able to expand the analyses by using data from the large GIANT meta-analyses $(n=123,864)$ [23]. However, this cannot be considered an independent replication, as eight of the studies that were part of the D-CarDia Collaboration were also included in GIANT. The F-statistic is used to measure the strength of an instrument, and an instrument that has a value greater than 10 is considered strong enough to use in IV analyses [49]. In our analyses, the F-statistic was greater than 200 for all instruments used due to our large sample size.

Combining large population-based studies from North America and Europe could lead to confounding by population stratification;

Table 2. Summary of the coefficients used for IV ratio analyses.

\begin{tabular}{|c|c|c|c|c|}
\hline \multirow[t]{2}{*}{ IV } & \multirow{2}{*}{$\begin{array}{l}\text { Allele Score with the } \\
\text { Intermediate Trait } \\
\text { Coefficient, \% }(95 \% \mathrm{CI})\end{array}$} & \multirow{2}{*}{$\begin{array}{l}\text { Allele Score with the Outcome } \\
\text { Coefficient, } \%(95 \% \mathrm{Cl})\end{array}$} & \multicolumn{2}{|l|}{ IV Ratio ${ }^{a}$} \\
\hline & & & Coefficient (95\% Cl) & $p$-Value \\
\hline BMI risk score & $0.14(0.12-0.16)$ & $-0.06(-0.1$ to -0.02$)$ & $-0.42(-0.71$ to -0.13$)$ & 0.005 \\
\hline Synthesis score & $-3.47(-3.90$ to -3.05$)$ & $0.01(-0.17$ to 0.20$)$ & $-0.00(-0.06$ to 0.05$)$ & 0.88 \\
\hline Metabolism score & $-5.38(-5.84$ to -4.93$)$ & $0.17(-0.02$ to 0.35$)$ & $-0.03(-0.06$ to 0.01$)$ & 0.08 \\
\hline
\end{tabular}

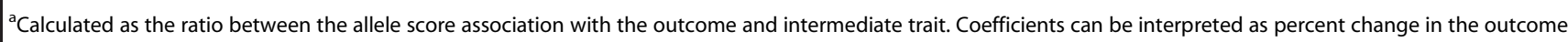
by percent change in the intermediate trait.

doi:10.1371/journal.pmed.1001383.t002 
Table 3. Results for the association between vitamin D SNPs/allele scores and BMI from the GIANT consortium.

\begin{tabular}{|c|c|c|c|}
\hline SNPs/Allele Scores & Gene Symbol & $\begin{array}{l}\text { Per Allele Change in BMI, } \\
\mathrm{kg} / \mathrm{m}^{2}(95 \% \mathrm{Cl})\end{array}$ & $p$-Value \\
\hline rs12785878 & DHCR7 & 0.001 ( -0.01 to 0.009$)$ & 0.78 \\
\hline rs10741657 & CYP2R1 & $-0.005(-0.004$ to 0.01$)$ & 0.30 \\
\hline Synthesis allele score (rs12785878+rs10741657) & $D H C R 7+C Y P 2 R 1$ & $-0.002(-0.009 \text { to } 0.005)^{\mathrm{a}}$ & 0.57 \\
\hline rs2282679 & GC & $0.001(-0.011$ to 0.010$)$ & 0.91 \\
\hline rs6013897 & CYP24A1 & $0.003(-0.008$ to 0.014$)$ & 0.61 \\
\hline Metabolism allele score (rs2282679+rs6013897) & $G C+C Y P 24 A 1$ & $0.002(-0.006 \text { to } 0.009)^{\mathrm{a}}$ & 0.67 \\
\hline
\end{tabular}

The GIANT meta-analyses consisted of 46 studies with up to 123,865 adults of European ancestry [23], including the 1958 British Birth Cohort, Framingham Heart study, Nurses' Health Study, Twins UK, UK Blood Services Common Control Collection, the Amish Family Osteoporosis Study, Health2000 GENMETS sub-sample, and Northern Finland Birth Cohort 1966, which were also part of the D-CarDia collaboration.

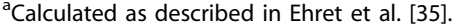

doi:10.1371/journal.pmed.1001383.t003

however, we adjusted for geographical variation/principal components in all analyses, which appeared adequate, as there was no evidence for heterogeneity by continent for the allele score metaanalyses. An important benefit of the MR approach is that it helps to overcome problems of confounding and reverse causality, which limit the ability to draw causal inferences in non-genetic observational studies [19,20]. However, it could be argued that as the biological function for some of the BMI SNPs is yet to be established, there could be alternative biological pathways explaining their association with BMI. Using multiple SNPs to index BMI, we were able to minimise the risk of pleiotropic effects, as the effects of alternative pathways reflected by individual SNPs would be expected to be strongly diluted when combined in a multi marker score [21,22].

In conclusion, we demonstrated that the association between BMI and lower 25(OH)D concentrations in Caucasian populations from North America and Europe can be seen across different age groups and in both men and women. We also show that higher BMI leads to lower vitamin D status, providing evidence for the role of obesity as a causal risk factor for the development of vitamin D deficiency. Together with the suggested increases in vitamin D requirements in obese individuals $[45,50]$, our study highlights the importance of monitoring and treating vitamin $\mathrm{D}$ deficiency as a means of alleviating the adverse influences of excess adiposity on health. Our findings suggest that population level interventions to reduce obesity would be expected to lead to a reduction in the prevalence of vitamin $\mathrm{D}$ deficiency.

\section{Supporting Information}

Figure S1 Vitamin D pathway showing the "synthesis" and "metabolism" indicators.

(TIF)

Figure S2 Meta-analysis of BMI allele score association with $B M I$ in collaborating studies $(n=32,391)$.

(TIF)

Figure S3 Meta-analysis of synthesis $(A)(n=35,873)$ and metabolism (B) $(n=38,191)$ allele score associations with $25(\mathrm{OH}) \mathrm{D}$ in collaborating studies.

(TIF)

Figure S4 Association of the 12 BMI-related SNPs with BMI.

(TIF)
Figure S5 Association of the four vitamin D SNPs with $25(\mathrm{OH})$ D.

(TIF)

Figure S6 Association of the 12 BMI-related SNPs with $25(\mathrm{OH})$ D.

(TIF)

Figure S7 Association of the four vitamin D SNPs with BMI.

(TIF)

Figure S8 Meta-analysis of synthesis allele score association with BMI stratified by mean BMI of collaborating studies $(n=36,553)$.

(TIF)

Figure S9 Power to detect an association between BMI and $25(\mathrm{OH}) D$ using genetic proxies in instrumental variable regression. The solid lines represent the power to detect the same effect size for a BMI association with $25(\mathrm{OH}) \mathrm{D}$ using BMI risk score (black line), 25(OH)D association with BMI using metabolism score (mid grey line), and 25(OH)D association with BMI using synthesis score (dark grey line). The dash lines represent the power to detect an effect size half that of the same coloured solid lines for the $25(\mathrm{OH}) \mathrm{D}$ association with BMI.

(TIF)

Table S1 Minor allele frequency (MAF) for the BMI and vitamin D-related SNPs.

(DOCX)

Text S1 STROBE statement-checklist of items. (DOC)

Text S2 Study details of the 21 participating cohorts and the funding information.

(DOCX)

\section{Acknowledgments}

We thank Alana Cavadino (UCL ICH) for technical assistance in formatting the figures.

Members of the Genetic Investigation of Anthropometric Traits (GIANT) Consortium include:

Elizabeth K Speliotes, Cristen J Willer, Sonja I Berndt, Keri L Monda, Gudmar Thorleifsson, Anne U Jackson, Hana Lango Allen, Cecilia M Lindgren, Jian'an Luan, Reedik Mägi, Joshua C Randall, Sailaja Vedantam, Thomas W Winkler, Lu Qi, Tsegaselassie Workalemahu, Iris M Heid, Valgerdur Steinthorsdottir, Heather M Stringham, Michael N 
Weedon, Eleanor Wheeler, Andrew R Wood, Teresa Ferreira, Robert J Weyant, Ayellet V Segrè, Karol Estrada, Liming Liang, James Nemesh, JuHyun Park, Stefan Gustafsson, Tuomas O Kilpeläinen, Jian Yang, Nabila Bouatia-Naji, Tõnu Esko, Mary F Feitosa, Zoltán Kutalik, Massimo Mangino, Soumya Raychaudhuri, Andre Scherag, Albert Vernon Smith, Ryan Welch, Jing Hua Zhao, Katja K Aben, Devin M Absher, Najaf Amin, Anna L Dixon, Eva Fisher, Nicole L Glazer, Michael E Goddard, Nancy L Heard-Costa, Volker Hoesel, Jouke-Jan Hottenga, Åsa Johansson, Toby Johnson, Shamika Ketkar, Claudia Lamina, Shengxu Li, Miriam F Moffatt, Richard H Myers, Narisu Narisu, John R B Perry, Marjolein J Peters, Michael Preuss, Samuli Ripatti, Fernando Rivadeneira, Camilla Sandholt, Laura J Scott, Nicholas J Timpson, Jonathan P Tyrer, Sophie van Wingerden, Richard M Watanabe, Charles C White, Fredrik Wiklund, Christina Barlassina, Daniel I Chasman, Matthew N Cooper, John-Olov Jansson, Robert W Lawrence, Niina Pellikka, Inga Prokopenko, Jianxin Shi, Elisabeth Thiering, Helene Alavere, Maria T S Alibrandi, Peter Almgren, Alice M Arnold, Thor Aspelund, Larry D Atwood, Beverley Balkau, Anthony J Balmforth, Amanda J Bennett, Yoav BenShlomo, Richard N Bergman, Sven Bergmann, Heike Biebermann, Alexandra I F Blakemore, Tanja Boes, Lori L Bonnycastle, Stefan R Bornstein, Morris J Brown, Thomas A Buchanan, Fabio Busonero, Harry Campbell, Francesco P Cappuccio, Christine Cavalcanti-Proença, Yii-Der Ida Chen, Chih-Mei Chen, Peter S Chines, Robert Clarke, Lachlan Coin, John Connell, Ian N M Day, Martin den Heijer, Jubao Duan, Shah Ebrahim, Paul Elliott, Roberto Elosua, Gudny Eiriksdottir, Michael R Erdos, Johan G Eriksson, Maurizio F Facheris, Stephan B Felix, Pamela Fischer-Posovszky, Aaron R Folsom, Nele Friedrich, Nelson B Freimer, Mao Fu, Stefan Gaget, Pablo V Gejman, Eco J C Geus, Christian Gieger, Anette P Gjesing, Anuj Goel, Philippe Goyette, Harald Grallert, Jürgen Gräßler, Danielle M Greenawalt, Christopher J Groves, Vilmundur Gudnason, Candace Guiducci, Anna-Liisa Hartikainen, Neelam Hassanali, Alistair S Hall, Aki S Havulinna, Caroline Hayward, Andrew C Heath, Christian Hengstenberg, Andrew A Hicks, Anke Hinney, Albert Hofman, Georg Homuth, Jennie Hui, Wilmar Igl, Carlos Iribarren, Bo Isomaa, Kevin B Jacobs, Ivonne Jarick, Elizabeth Jewell, Ulrich John, Torben Jørgensen, Pekka Jousilahti, Antti Jula, Marika Kaakinen, Eero Kajantie, Lee M Kaplan, Sekar Kathiresan, Johannes Kettunen, Leena Kinnunen, Joshua W Knowles, Ivana Kolcic, Inke R König, Seppo Koskinen, Peter Kovacs, Johanna Kuusisto, Peter Kraft, Kirsti Kvaløy, Jaana Laitinen, Olivier Lantieri, Chiara Lanzani, Lenore J Launer, Cecile Lecoeur, Terho Lehtimäki, Guillaume Lettre, Jianjun Liu, Marja-Liisa Lokki, Mattias Lorentzon, Robert N Luben, Barbara Ludwig, Paolo Manunta, Diana Marek, Michel Marre, Nicholas G Martin, Wendy L McArdle, Anne McCarthy, Barbara McKnight, Thomas Meitinger, Olle Melander, David Meyre, Kristian Midthjell, Grant W Montgomery, Mario A Morken, Andrew P Morris, Rosanda Mulic, Julius S Ngwa, Mari Nelis, Matt J Neville, Dale R Nyholt, Christopher J O'Donnell, Stephen O'Rahilly, Ken K Ong, Ben Oostra, Guillaume Paré, Alex N Parker, Markus Perola, Irene Pichler, Kirsi H Pietiläinen, Carl G P Platou, Ozren Polasek, Anneli Pouta, Suzanne Rafelt, Olli Raitakari, Nigel W Rayner, Martin Ridderstråle, Winfried Rief, Aimo Ruokonen, Neil R Robertson, Peter Rzehak, Veikko Salomaa, Alan R Sanders, Manjinder S Sandhu, Serena Sanna, Jouko Saramies, Markku J Savolainen, Susann Scherag, Sabine Schipf, Stefan Schreiber, Heribert Schunkert, Kaisa Silander, Juha Sinisalo, David S Siscovick, Jan H Smit, Nicole Soranzo, Ulla Sovio, Jonathan Stephens, Ida Surakka, Amy J Swift, Mari-Liis Tammesoo, JeanClaude Tardif, Maris Teder-Laving, Tanya M Teslovich, John R Thompson, Brian Thomson, Anke Tönjes, Tiinamaija Tuomi, Joyce B J van Meurs, Gert-Jan van Ommen, Vincent Vatin, Jorma Viikari, Sophie
Visvikis-Siest, Veronique Vitart, Carla I G Vogel, Benjamin F Voight, Lindsay L Waite, Henri Wallaschofski, G Bragi Walters, Elisabeth Widen, Susanna Wiegand, Sarah H Wild, Gonneke Willemsen, Daniel R Witte, Jacqueline $\mathrm{C}$ Witteman, Jianfeng Xu, Qunyuan Zhang, Lina Zgaga, Andreas Ziegler, Paavo Zitting, John P Beilby, I Sadaf Farooqi, Johannes Hebebrand, Heikki V Huikuri,Alan L James, Mika Kähönen, Douglas F Levinson, Fabio Macciardi, Markku S Nieminen, Claes Ohlsson, Lyle J Palmer, Paul M Ridker, Michael Stumvoll, Jacques S Beckmann, Heiner Boeing, Eric Boerwinkle, Dorret I Boomsma, Mark J Caulfield, Stephen J Chanock, Francis S Collins, L Adrienne Cupples, George Davey Smith, Jeanette Erdmann, Philippe Froguel, Henrik Grönberg, Ulf Gyllensten, Per Hall, Torben Hansen, Tamara B Harris, Andrew T Hattersley, Richard B Hayes, Joachim Heinrich, Frank B Hu, Kristian Hveem, Thomas Illig, Marjo-Riitta Jarvelin, Jaakko Kaprio, Fredrik Karpe, Kay-Tee Khaw, Lambertus A Kiemeney, Heiko Krude, Markku Laakso, Debbie A Lawlor, Andres Metspalu, Patricia B Munroe, Willem H Ouwehand, Oluf Pedersen, Brenda W Penninx, Annette Peters, Peter P Pramstaller, Thomas Quertermous, Thomas Reinehr, Aila Rissanen, Igor Rudan, Nilesh J Samani, Peter E H Schwarz, Alan R Shuldiner, Timothy D Spector, Jaakko Tuomilehto, Manuela Uda, André Uitterlinden, Timo T Valle, Martin Wabitsch, Gérard Waeber, Nicholas J Wareham,Hugh Watkins, James F Wilson, Alan F Wright, M Carola Zillikens, Nilanjan Chatterjee, Steven A McCarroll, Shaun Purcell, Eric E Schadt, Peter M Visscher, Themistocles L Assimes, Ingrid B Borecki, Panos Deloukas, Caroline S Fox, Leif C Groop, Talin Haritunians, David J Hunter, Robert C Kaplan, Karen L Mohlke, Jeffrey R O'Connell, Leena Peltonen, David Schlessinger, David P Strachan, Cornelia M van Duijn, H-Erich Wichmann, Timothy M Frayling, Unnur Thorsteinsdottir, Gonçalo R Abecasis, Inês Barroso, Michael Boehnke, Kari Stefansson, Kari E North, Mark I McCarthy, Joel N Hirschhorn, Erik Ingelsson and Ruth J F Loos.

\section{Author Contributions}

Conceived and designed the experiments: KSV DJB MM OR JV BDM EAM NJW TM TL WHO CG TJW MRJJCW ADH EH. Performed the experiments: KSV DJB CL ET SP LTH JDC KHL ZD RL ARW KM LZ LMY JD MCK KJ VS BDM EAS DKH TL PK CG RJFL JCW ADH EH. Analyzed the data: KSV DJB CL ET SP LTH JDC KHL ZD RL ARW KM LZ LMY JD MCK KJ VS BDM EAS DKH TL PK CG RJFL JCW ADH EH. Contributed reagents/materials/analysis tools: KSV DJB SP LTH KM LMY MM JD MK NA OR JV SBK HM EI LB LL VS BDM KH EAS DKH TL PK CC WM RJFL CP MRJ JCW ADH EH. Wrote the first draft of the manuscript: KSV DJB EH. Contributed to the writing of the manuscript: KSV DJB EH. ICMJE criteria for authorship read and met: KSV DJB CL ET SP LTH JDC ZD RL DKH ARW KM LV LZ LMY MM JD MK MEK KJ NA OR JV KKL LF HM EI LB LL ML VS HC MD BDM KHH AP ALH EAS ET AJ NJW CO TMF SBK TDS JBR TL WHO PK CG WM CP RJFL TJW MRJ JCW ADH EH. Agree with manuscript results and conclusions: KSV DJB CL ET SP LTH JDC ZD RL DKH ARW KM LV LZ LMY MM JD MK MEK KJ NA OR JV KKL LF HM EI LB LL ML VS HC MD BDM KHH AP ALH EAS ET AJ NJW CO TMF SBK TDS JBR TL WHO PK CG WM CP RJFL TJW MRJ JCW ADH EH. Obtained the data: KSV DJB SP LTH ZD RL KM LV MM MK MCK KJ NA OR JV SBK LF HM EI LB LL ML VS HC MD BDM AP AH ET NJW CO TMF DKH JBR TL WHO PK CC WM CP ADH MRJ EH. Provided the administrative, technical, or material support: KSV DJB KM MK OR JV SBK LF HM EI LL VS TMF DKH TL GP EH. Supervised the study: JD OR JV SBK HC MD BDM EAS AJ ET TS TMF JBR TL TJW MRJ JCW ADH EH.

\section{References}

1. Baskin ML, Ard J, Franklin F, Allison DB (2005) Prevalence of obesity in the United States. Obes Rev 6: 5-7.

2. Ogden CL, Carroll MD, Curtin LR, Lamb MM, Flegal KM (2010) Prevalence of high body mass index in US children and adolescents, 2007-2008. JAMA 303: 242-249.

3. Berghofer A, Pischon T, Reinhold T, Apovian CM, Sharma AM, et al. (2008) Obesity prevalence from a European perspective: a systematic review. BMC Public Health 8: 200.
4. Zheng W, McLerran DF, Rolland B, Zhang X, Inoue M, et al. (2011) Association between body-mass index and risk of death in more than 1 million Asians. N Engl J Med 364: 719-729.

5. Flegal KM, Carroll MD, Kit BK, Ogden CL (2012) Prevalence of obesity and trends in the distribution of body mass index among US adults, 1999-2010. JAMA 307: 491-497.

6. Vimaleswaran KS, Loos RJ (2010) Progress in the genetics of common obesity and type 2 diabetes. Expert Rev Mol Med 12: e7. 
7. Ginde AA, Liu MC, Camargo CA, Jr. (2009) Demographic differences and trends of vitamin D insufficiency in the US population, 1988-2004. Arch Intern Med 169: 626-632.

8. Lanham-New SA, Buttriss JL, Miles LM, Ashwell M, Berry JL, et al. (2011) Proceedings of the Rank Forum on vitamin D. Br J Nutr 105: 144-156.

9. Hyppönen E, Power C (2007) Hypovitaminosis D in British adults at age 45 y: nationwide cohort study of dietary and lifestyle predictors. Am J Clin Nutr 85: 860-868.

10. Earthman CP, Beckman LM, Masodkar K, Sibley SD (2012) The link between obesity and low circulating 25-hydroxyvitamin D concentrations: considerations and implications. Int J Obes (Lond) 36: 387-396.

11. Shi H, Norman AW, Okamura WH, Sen A, Zemel MB (2001) lalpha,25Dihydroxyvitamin D3 modulates human adipocyte metabolism via nongenomic action. Faseb J 15: 2751-2753.

12. Fassina G, Maragno I, Dorigo P, Contessa AR (1969) Effect of vitamin D2 on hormone-stimulated lipolysis in vitro. Eur J Pharmacol 5: 286-290.

13. Sneve M, Figenschau Y, Jorde R (2008) Supplementation with cholecalciferol does not result in weight reduction in overweight and obese subjects. Eur J Endocrinol 159: 675-684.

14. Zittermann A, Frisch S, Berthold HK, Gotting C, Kuhn J, et al. (2009) Vitamin D supplementation enhances the beneficial effects of weight loss on cardiovascular disease risk markers. Am J Clin Nutr 89: 1321-1327.

15. Salehpour A, Shidfar F, Hosseinpanah F, Vafa M, Razaghi M, et al. (2012) Vitamin D3 and the risk of CVD in overweight and obese women: a randomised controlled trial. Br J Nutr: 1-8.

16. Soares MJ, Murhadi LL, Kurpad AV, Chan She Ping-Delfos WL, Piers LS (2012) Mechanistic roles for calcium and vitamin D in the regulation of body weight. Obes Rev 13: 592-605.

17. Foss YJ (2009) Vitamin D deficiency is the cause of common obesity. Med Hypotheses 72: 314-321.

18. Wortsman J, Matsuoka LY, Chen TC, Lu Z, Holick MF (2000) Decreased bioavailability of vitamin D in obesity. Am J Clin Nutr 72: 690-693.

19. Davey Smith G, Ebrahim S (2003) 'Mendelian randomization': can genetic epidemiology contribute to understanding environmental determinants of disease? Int J Epidemiol 32: 1-22.

20. Lawlor DA, Harbord RM, Sterne JA, Timpson N, Davey Smith G (2008) Mendelian randomization: using genes as instruments for making causal inferences in epidemiology. Stat Med 27: 1133-1163.

21. Davey Smith G (2011) Random allocation in observational data: how small but robust effects could facilitate hypothesis-free causal inference. Epidemiology 22: 460-463; discussion 467-468.

22. Palmer TM, Lawlor DA, Harbord RM, Sheehan NA, Tobias JH, et al. (2012) Using multiple genetic variants as instrumental variables for modifiable risk factors. Stat Methods Med Res 21: 223-242.

23. Speliotes EK, Willer CJ, Berndt SI, Monda KL, Thorleifsson G, et al. (2010) Association analyses of 249,796 individuals reveal 18 new loci associated with body mass index. Nat Genet 42: 937-948.

24. Li S, Zhao JH, Luan J, Luben RN, Rodwell SA, et al. (2010) Cumulative effects and predictive value of common obesity-susceptibility variants identified by genome-wide association studies. Am J Clin Nutr 91: 184-190.

25. Loos RJ, Lindgren CM, Li S, Wheeler E, Zhao JH, et al. (2008) Common variants near MC4R are associated with fat mass, weight and risk of obesity. Nat Genet 40: 768-775.

26. Thorleifsson G, Walters GB, Gudbjartsson DF, Steinthorsdottir V, Sulem P, et al. (2009) Genome-wide association yields new sequence variants at seven loci that associate with measures of obesity. Nat Genet 41: 18-24.

27. Wang TJ, Zhang F, Richards JB, Kestenbaum B, van Meurs JB, et al. (2010) Common genetic determinants of vitamin D insufficiency: a genome-wide association study. Lancet 376: 180-188.

28. Zheng J, Li Y, Abecasis GR, Scheet P (2011) A comparison of approaches to account for uncertainty in analysis of imputed genotypes. Genet Epidemiol 35: $102-110$.
29. Cole TJ (2000) Sympercents: symmetric percentage differences on the $100 \log (\mathrm{e})$ scale simplify the presentation of log transformed data. Stat Med 19: 3109-3125.

30. Lin X, Song K, Lim N, Yuan X, Johnson T, et al. (2009) Risk prediction of prevalent diabetes in a Swiss population using a weighted genetic score-the CoLaus Study. Diabetologia 52: 600-608.

31. Pierce BL, Ahsan H, Vanderweele TJ (2011) Power and instrument strength requirements for Mendelian randomization studies using multiple genetic variants. Int J Epidemiol 40: 740-752.

32. Berry DJ, Vimaleswaran KS, Whittaker JC, Hingorani AD, Hypponen E (2012) Evaluation of genetic markers as instruments for mendelian randomization studies on vitamin D. PLoS One 7: e37465. doi:10.1371/journal.pone.0037465

33. Chun RF, Lauridsen AL, Suon L, Zella LA, Pike JW, et al. (2010) Vitamin Dbinding protein directs monocyte responses to 25-hydroxy- and 1,25dihydroxyvitamin D. J Clin Endocrinol Metab 95: 3368-3376.

34. Rice JA (1995) Expected values. Mathematical statistics and data analysis. 2nd edition. Pacific Grove (California): Duxbury Press.

35. Ehret GB, Munroe PB, Rice KM, Bochud M, Johnson AD, et al. (2011) Genetic variants in novel pathways influence blood pressure and cardiovascular disease risk. Nature 478: 103-109.

36. Thomas DC, Lawlor DA, Thompson JR (2007) Re: Estimation of bias in nongenetic observational studies using "Mendelian triangulation" by Bautista et al. Ann Epidemiol 17: 511-513.

37. White IR (2009) Multivariate random-effects meta-analysis. The Stata Journal 9: 40-56.

38. Borenstein M (2009) Introduction to meta-analysis. Chichester: John Wiley \& Sons. xxviii.

39. StataCorp (2011). Stata Statistical Software: Release 12: College Station (Texas): StataCorp LP.

40. Jorde R, Sneve M, Emaus N, Figenschau Y, Grimnes G (2010) Cross-sectional and longitudinal relation between serum 25-hydroxyvitamin $\mathrm{D}$ and body mass index: the Tromso study. Eur J Nutr 49: 401-407.

41. Lee P, Greenfield JR, Seibel MJ, Eisman JA, Center JR (2009) Adequacy of vitamin $\mathrm{D}$ replacement in severe deficiency is dependent on body mass index. Am J Med 122: 1056-1060.

42. Bassett DR, Jr., Pucher J, Buehler R, Thompson DL, Crouter SE (2008) Walking, cycling, and obesity rates in Europe, North America, and Australia. J Phys Act Health 5: 795-814.

43. Dorjgochoo T, Shi J, Gao YT, Long J, Delahanty R, et al. (2012) Genetic variants in vitamin $\mathrm{D}$ metabolism-related genes and body mass index: analysis of genome-wide scan data of approximately 7000 Chinese women. Int J Obes (Lond) 36: 1252-1255.

44. Drincic AT, Armas LA, Van Diest EE, Heaney RP (2012) Volumetric dilution, rather than sequestration best explains the low vitamin D status of obesity. Obesity (Silver Spring) 20: 1444-1448.

45. Bell NH, Epstein S, Greene A, Shary J, Oexmann MJ, et al. (1985) Evidence for alteration of the vitamin D-endocrine system in obese subjects. J Clin Invest 76: 370-373.

46. Holick MF (2007) Vitamin D deficiency. N Engl J Med 357: 266-281.

47. Hyppönen E, Berry D, Cortina-Borja M, Power C (2010) 25-Hydroxyvitamin D and pre-clinical alterations in inflammatory and hemostatic markers: a cross sectional analysis in the 1958 British Birth Cohort. PLoS One 5: e10801. doi:10.1371/journal.pone.0010801

48. Burgess S, Thompson SG, Andrews G, Samani NJ, Hall A, et al. (2010) Bayesian methods for meta-analysis of causal relationships estimated using genetic instrumental variables. Stat Med 29: 1298-1311.

49. Staiger D, Stock JH (1997) Instrumental variables regression with weak instruments. Econometrica 65: 557-586.

50. Huh SY, Gordon CM (2008) Vitamin D deficiency in children and adolescents: epidemiology, impact and treatment. Rev Endocr Metab Disord 9: 161-170. 


\section{Editors' Summary}

Background. Obesity-having an unhealthy amount of body fat-is increasing worldwide. In the US, for example, a third of the adult population is now obese. Obesity is defined as having a body mass index (BMI, an indicator of body fat calculated by dividing a person's weight in kilograms by their height in meters squared) of more than $30.0 \mathrm{~kg} / \mathrm{m}^{2}$. Although there is a genetic contribution to obesity, people generally become obese by consuming food and drink that contains more energy than they need for their daily activities. Thus, obesity can be prevented by having a healthy diet and exercising regularly. Compared to people with a healthy weight, obese individuals have an increased risk of developing diabetes, heart disease and stroke, and tend to die younger. They also have a higher risk of vitamin D deficiency, another increasingly common public health concern. Vitamin D, which is essential for healthy bones as well as other functions, is made in the skin after exposure to sunlight but can also be obtained through the diet and through supplements.

Why Was This Study Done? Observational studies cannot prove that obesity causes vitamin $D$ deficiency because obese individuals may share other characteristics that reduce their circulating 25-hydroxy vitamin D [25(OH)D] levels (referred to as confounding). Moreover, observational studies cannot indicate whether the larger vitamin D storage capacity of obese individuals (vitamin $D$ is stored in fatty tissues) lowers their $25(\mathrm{OH}) \mathrm{D}$ levels or whether $25(\mathrm{OH}) \mathrm{D}$ levels influence fat accumulation (reverse causation). If obesity causes vitamin D deficiency, monitoring and treating vitamin $D$ deficiency might alleviate some of the adverse health effects of obesity. Conversely, if low vitamin D levels cause obesity, encouraging people to take vitamin D supplements might help to control the obesity epidemic. Here, the researchers use bi-directional "Mendelian randomization" to examine the direction and causality of the relationship between $\mathrm{BMI}$ and $25(\mathrm{OH}) \mathrm{D}$. In Mendelian randomization, causality is inferred from associations between genetic variants that mimic the influence of a modifiable environmental exposure and the outcome of interest. Because gene variants do not change over time and are inherited randomly, they are not prone to confounding and are free from reverse causation. Thus, if a lower vitamin $D$ status leads to obesity, genetic variants associated with lower 25(OH)D concentrations should be associated with higher BMI, and if obesity leads to a lower vitamin D status, then genetic variants associated with higher BMI should be associated with lower $25(\mathrm{OH}) \mathrm{D}$ concentrations.

What Did the Researchers Do and Find? The researchers created a "BMI allele score" based on 12 BMl-related gene variants and two "25(OH)D allele scores," which are based on gene variants that affect either $25(\mathrm{OH}) \mathrm{D}$ synthesis or breakdown. Using information on up to 42,024 participants from 21 studies, the researchers showed that the BMI allele score was associated with both BMI and with $25(\mathrm{OH}) \mathrm{D}$ levels among the study participants. Based on this information, they calculated that each $10 \%$ increase in BMI will lead to a
$4.2 \%$ decrease in $25(\mathrm{OH}) \mathrm{D}$ concentrations. By contrast, although both $25(\mathrm{OH}) \mathrm{D}$ allele scores were strongly associated with $25(\mathrm{OH}) \mathrm{D}$ levels, neither score was associated with BMI. This lack of an association between 25(OH)D allele scores and obesity was confirmed using data from more than 100,000 individuals involved in 46 studies that has been collected by the GIANT (Genetic Investigation of Anthropometric Traits) consortium.

What Do These Findings Mean? These findings suggest that a higher BMI leads to a lower vitamin D status whereas any effects of low vitamin D status on BMI are likely to be small. That is, these findings provide evidence for obesity as a causal factor in the development of vitamin D deficiency but not for vitamin $D$ deficiency as a causal factor in the development of obesity. These findings suggest that population-level interventions to reduce obesity should lead to a reduction in the prevalence of vitamin D deficiency and highlight the importance of monitoring and treating vitamin $D$ deficiency as a means of alleviating the adverse influences of obesity on health.

Additional Information. Please access these Web sites via the online version of this summary at http://dx.doi.org/10. 1371/journal.pmed.1001383.

- The US Centers for Disease Control and Prevention provides information on all aspects of overweight and obesity (in English and Spanish); a data brief provides information about the vitamin D status of the US population

- The World Health Organization provides information on obesity (in several languages)

- The UK National Health Service Choices website provides detailed information about obesity and a link to a personal story about losing weight; it also provides information about vitamin D

- The International Obesity Taskforce provides information about the global obesity epidemic

- The US Department of Agriculture's ChooseMyPlate.gov website provides a personal healthy eating plan; the Weight-control Information Network is an information service provided for the general public and health professionals by the US National Institute of Diabetes and Digestive and Kidney Diseases (in English and Spanish)

- The US Office of Dietary Supplements provides information about vitamin D (in English and Spanish)

- MedlinePlus has links to further information about obesity and about vitamin D (in English and Spanish)

- Wikipedia has a page on Mendelian randomization (note: Wikipedia is a free online encyclopedia that anyone can edit; available in several languages)

- Overview and details of the collaborative large-scale genetic association study (D-CarDia) provide information about vitamin $\mathrm{D}$ and the risk of cardiovascular disease, diabetes and related traits 